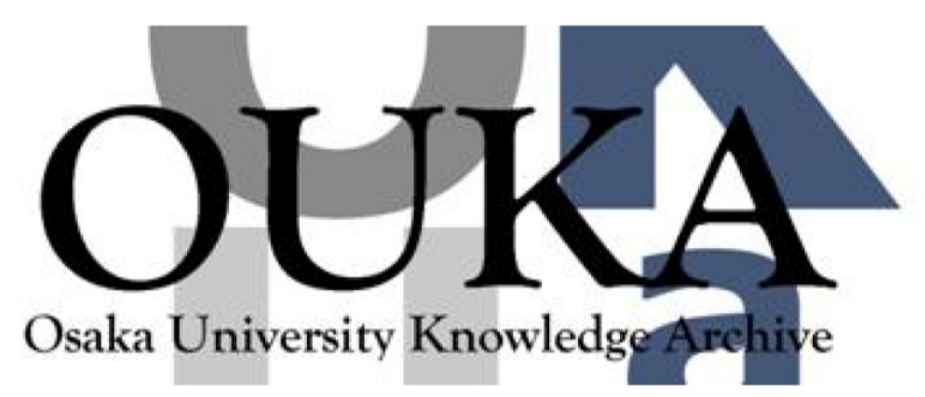

\begin{tabular}{|c|c|}
\hline Title & $\begin{array}{l}\text { Spin separation and spin Hall effect in quantum } \\
\text { wires due to lateral-confinement-induced spin- } \\
\text { orbit-coupling }\end{array}$ \\
\hline Author (s) & Hattori, Kiminori; Okamoto, Hiroaki \\
\hline Citation & PHYSICAL REVIEW B. 74(15) p. 155321-1-p. 155321-6 \\
\hline Issue Date & $2006-10-25$ \\
\hline oaire:version & VoR \\
\hline URL & https://hdl. handle. net/11094/3376 \\
\hline rights & $\begin{array}{l}\text { Hattori, Kiminori, Okamoto, Hiroaki, Physical } \\
\text { Review B, 74,15, 155321, 2006-10-25. } \\
\text { "Copyright } 2006 \text { by the American Physical } \\
\text { Society." }\end{array}$ \\
\hline Note & \\
\hline
\end{tabular}

Osaka University Knowledge Archive : OUKA

https://ir. Library. osaka-u. ac. jp/

Osaka University 


\title{
Spin separation and spin Hall effect in quantum wires due to lateral-confinement-induced spin-orbit-coupling
}

\author{
Kiminori Hattori and Hiroaki Okamoto \\ Department of Systems Innovation, Graduate School of Engineering Science, Osaka University, Toyonaka, Osaka 560-8531, Japan
}

(Received 1 June 2006; revised manuscript received 26 September 2006; published 25 October 2006)

\begin{abstract}
We show that the spin-orbit coupling in two-dimensional (2D) electron systems with a potential gradient parallel to the 2D plane exerts a spin-dependent transverse force on moving electrons while conserving their spins. Due to the spin conservation, the standard continuity equation holds between spin density and spin current. Using numerical calculations based on the nonequilibrium Green's function formalism, we demonstrate for crossed quantum wires with a harmonic confining potential in the ballistic limit that transverse spin separation and spin Hall current are generated in response to a longitudinal charge current.
\end{abstract}

DOI: 10.1103/PhysRevB.74.155321

PACS number(s): 73.23.Ad, 71.70.Ej, 72.25.Dc

Spin-orbit (SO) coupling brings about a number of intriguing phenomena, ${ }^{1}$ one of which is the spin Hall effect, i.e., the generation of a transverse spin flux due to the electric field applied to an infinitely-large homogeneous system or the electrochemical potential difference between the electrodes attached to a finite-size mesoscopic system. ${ }^{2-13}$ The possibility to manipulate spin current by a purely electrical means attracts a great deal of interest in the field of spintronics since it enables spin injection to and spin polarization in conventional semiconductors without ferromagnetic materials and magnetic fields. To date, various classes of spin Hall effects have been discovered in different physical systems and studied extensively. In the case of quantum heterostructures of narrow gap semiconductors, a major contribution to the SO coupling may originate intrinsically from its confining potential. ${ }^{14}$ The spin Hall effect in two-dimensional (2D) electron systems exploits the Rashba SO coupling due to an asymmetry in quantum well potential that confines the electron gas. ${ }^{6}$ As an extension of Ehrenfest's theorem in quantum mechanics, the SO coupling may generate a spin-dependent transverse force on moving electrons. ${ }^{15-19}$ This force tends to separate different spins in the transverse direction as a response to the longitudinal charge current, giving a qualitative explanation for the Rashba spin Hall effect. In the presence of Rashba SO coupling, however, electron spin (particularly its out-of-plane projection) is not conserved and, hence, the usual continuity equation fails to describe the spin transport. This makes the spin transport phenomena in this system rather complicated.

In this paper, we discuss the possibility of spin Hall effect in quantum wires (QWs) through the analysis of the SO coupling in a $2 \mathrm{D}$ electron system with an in-plane potential gradient. A SO coupling of this kind generates a spin-dependent force on moving electrons while conserving their spins. The standard continuity equation for spin density and spin current is naturally established because of spin conservation. We investigate the spin distribution and the spin transport in the ballistic QWs with a harmonic confining potential, and demonstrate the existence of a spin Hall current in the crossed QW geometry, using numerical calculations based on nonequilibrium Green's function formalism. ${ }^{20,21}$ The occurrence of a spin Hall effect in a harmonic QW system was suggested previously. ${ }^{22}$ The four-terminal junction considered in this study differs from the previous model assuming weaklycoupled spin current probes.

The SO interaction is generally described by the Hamiltonian $H_{\mathrm{SO}}=(\alpha / \hbar) \nabla U \cdot(\boldsymbol{\sigma} \times \mathbf{p})$, where $\mathbf{p}$ is the momentum operator, $\boldsymbol{\sigma}$ is the vector of Pauli matrices, and $\alpha$ is the SO coupling parameter having a dimension of length squared. We adapt the general form to the strictly $2 \mathrm{D}$ case, where the degree of freedom of motion in the $z$ direction is frozen out, and the potential energy $U(\mathbf{r})$ depends only on $x$ and $y$ coordinates. Then $H_{\text {SO }}$ takes the following form:

$$
H_{\mathrm{SO}}=\frac{\alpha}{\hbar}\left(u_{y} p_{x}-u_{x} p_{y}\right) \sigma_{z},
$$

where $u_{x, y}=\mathbf{e}_{x, y} \cdot \nabla U$ with the unit vectors $\mathbf{e}_{x, y}$ on the $x y$ plane. The reduced Hamiltonian commutes with the spin operator $S_{z}=(\hbar / 2) \sigma_{z}$, and, hence, conserves spin. This crucially differs from the Rashba SO coupling associated with $u_{z},{ }^{14}$ for which $H_{\mathrm{SO}}=(\alpha / \hbar) u_{z}\left(\sigma_{x} p_{y}-\sigma_{y} p_{x}\right)$, so that $\left[S_{z}, H_{\mathrm{SO}}\right] \neq 0$ unless $\mathbf{p}=0$ and spin precession takes place around the vector $\mathbf{p} \times \mathbf{e}_{z}$ in the course of electron transport. It may be worth mentioning that the spin-conserving SO interactions are also the basis of the quantum spin Hall effects discovered recently. ${ }^{11-13}$ Specifically, one of them, which stems from a strain gradient formed in conventional semiconductors, ${ }^{13}$ is apparently similar to Eq. (1).

The total Hamiltonian can be written as

$$
H=\frac{\mathbf{p}^{2}}{2 m}+U+\frac{\alpha}{\hbar}\left(u_{y} p_{x}-u_{x} p_{y}\right) \sigma_{z},
$$

including the kinetic energy term $\mathbf{p}^{2} / 2 m$ with the electron mass $m$. Using the Heisenberg equation, the kinetic velocity operator is formulated as $\mathbf{v}=[\mathbf{r}, H] / i \hbar=\mathbf{v}_{0}+\mathbf{v}_{\mathrm{SO}}$, where $\mathbf{v}_{0}=\mathbf{p} / m$ is the canonical velocity and $\mathbf{v}_{\mathrm{SO}}$ $=(\alpha / \hbar)\left(\mathbf{e}_{x} u_{y}-\mathbf{e}_{y} u_{x}\right) \sigma_{z}$ is the spin-dependent anomalous velocity due to the SO coupling. The same procedure also applies to introducing the force operator $\mathbf{F}$ as the time derivative of kinetic momentum operator $m \mathbf{v}$. For instance, a harmonic potential $U(x)=m \omega^{2} x^{2} / 2$ gives the operator $\mathbf{F}=\mathbf{F}_{0}+\mathbf{F}_{\text {SO }}$ consisting of two components, $\mathbf{F}_{0}=-m \omega^{2} x \mathbf{e}_{x}$ and $\mathbf{F}_{\mathrm{SO}}=\left(\alpha / l^{2}\right) \omega \mathbf{p} \times \mathbf{e}_{z} \sigma_{z}$, where $l=\sqrt{\hbar / m \omega}$ is the characteristic length scale of the confinement. The second term, $\mathbf{F}_{\mathrm{SO}}$, cor- 
responds to the spin-dependent SO coupling force, which is a purely quantum quantity and has no classical counterpart. This force acts on moving electrons and tends to deflect a spin- $\uparrow$ electron to the right and a spin- $\downarrow$ electron to the left. For simplicity, the present analysis neglects the anomalous contribution to the position operator due to the Berry gauge potential $\mathbf{A}=\left(\alpha / \hbar^{2}\right)(\boldsymbol{\sigma} \times \mathbf{p})$ in the momentum space. ${ }^{18,19}$ Taking this contribution into account to first order in $\alpha$ derives the anomalous velocity exactly twice as large as the $\mathbf{v}_{\mathrm{SO}}$ written above, as well as the additional term $-\left(\alpha / l^{2}\right) \omega p_{x} \sigma_{z} \mathbf{e}_{y}$ in the expression of the SO coupling force for $U(x)$ $=m \omega^{2} x^{2} / 2$. However, the correction is not substantial for the spin Hall effect in harmonic QWs discussed below, since the extra force is parallel to the wire orientation.

The Schrödinger equation $i \hbar \partial \psi / \partial t=H \psi$ in terms of the fermion field operator $\psi$ leads to the standard sourceless continuity equation

$$
\frac{\partial}{\partial t} \rho_{s}+\nabla \cdot \mathbf{J}_{s}=0
$$

relating the spin density operator $\rho_{s}=\psi{ }_{z} \psi$ and the spin current density operator $\mathbf{J}_{s}=\operatorname{Re} \psi^{\dagger} S_{z} \mathbf{v} \psi$, where $\operatorname{Re} A$ is defined as $\left(A+A^{\dagger}\right) / 2$. The absence of the spin torque contribution, which appears inevitably as a source (or sink) term in the case of the Rashba SO coupling, ${ }^{23-25}$ is simply due to the fact that the Hamiltonian considered here is diagonal in the spin basis. Actually, the standard continuity equation holds with respect to individual spinor components.

A typical physical system described by Eq. (2) is a QW. ${ }^{26}$ We consider the quasi-1D system formed when a 2D electron gas confined in the $z$ direction by a square potential is further subject to lateral confinement. When the lateral confining potential is parabolic, $U(x)=m \omega^{2} x^{2} / 2,{ }^{27-29}$ the effective Hamiltonian in each spin state can be expressed for a weak SO coupling such that $\alpha^{2} / l^{4} \ll 1$ as

$$
H_{\sigma}=\frac{\mathbf{p}^{2}}{2 m}+\frac{1}{2} m \omega^{2}\left(x-\frac{\alpha}{\hbar} p_{y} \sigma\right)^{2},
$$

where $\sigma= \pm 1$ for spin- $\uparrow$ and $\downarrow$ states, respectively. This system makes up 1D subbands with the energy

$$
\varepsilon_{n k}=\hbar \omega\left(n+\frac{1}{2}\right)+\frac{\hbar^{2} k^{2}}{2 m}
$$

for both spins, where $n(=0,1,2, \ldots)$ represents the subband index, and $k$ stands for the propagation wave vector along $y$. The corresponding eigenfunction for spin $\sigma$,

$$
\varphi_{n k \sigma}(x, y)=\frac{1}{\sqrt{L}} \exp (i k y) \phi_{n}(x-\alpha k \sigma),
$$

is spatially shifted by an amount $\alpha k \sigma$, where $\phi_{n}(x)$ is the $n$th eigenfunction of the 1D harmonic oscillator. The displacement is quantitatively interpreted in terms of the SO coupling force. Thus, moving electrons with opposite spins experience opposite SO coupling forces, yielding opposite signs of displacements. A nonequilibrium spin separation in the transverse direction will therefore be generated inside the $\mathrm{QW}$ in the presence of a charge current in the longitudinal

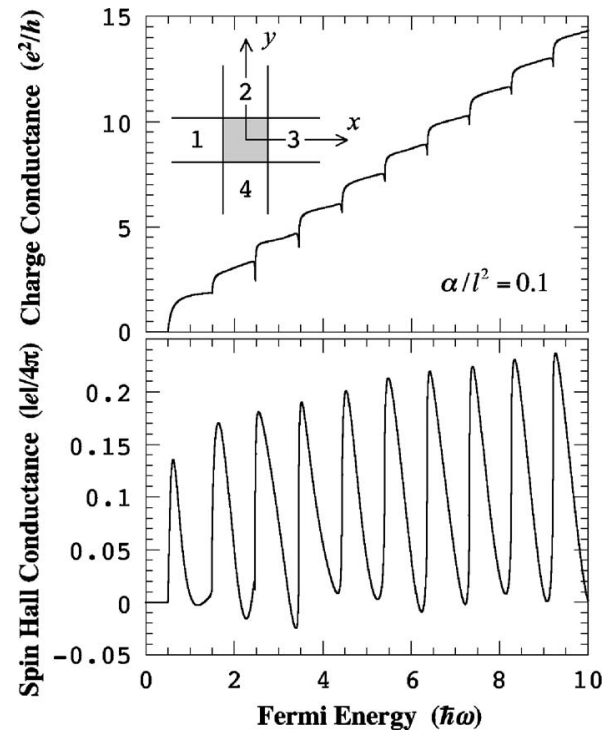

FIG. 1. Longitudinal charge conductance $G_{\mathrm{C}}$ and spin Hall conductance $G_{\mathrm{SH}}$ calculated as a function of Fermi energy $E$. In the calculation, the system size, and the SO coupling strength were set at $W=60 a$ and $\alpha / l^{2}=0.1$, respectively. Inset is a schematic view of the four-terminal junction of QWs.

direction. ${ }^{30}$ What is also suggested is that the transverse spin current flows as a response to the longitudinal charge current, when spin current probes are properly attached to the system.

In this study the spin Hall effect has been examined for a four-terminal junction of harmonic QWs by means of numerical calculations. The model system is shown in the inset of Fig. 1. Four semi-infinite QWs with an equal width $W$ labeled by $\lambda=1,2,3,4$ join at right angles. The confining potential in the interior of QWs running along the $x$ and $y$ axes was chosen as $U(\mathbf{r})=m \omega^{2} x^{2} / 2$ for $y^{2}>x^{2}$ and $m \omega^{2} y^{2} / 2$ for $x^{2}>y^{2} \cdot{ }^{31}$ Note that the model potential $U(\mathbf{r})$ produces the SO coupling force expressed as $\mathbf{F}_{\mathrm{SO}}=\left(\alpha / l^{2}\right) \omega \mathbf{p} \times \mathbf{e}_{z} \sigma_{z}$ everywhere, except on diagonal lines $x= \pm y$. For computational convenience, the hard wall potentials that define the finite width $\mathrm{W}$ are also assumed outside the crossed QWs. In the tight-binding approximation on a square lattice with lattice spacing $a$, the Hamiltonian of each spin subsystem is formulated as

$$
H_{\sigma}=\sum_{\mathbf{r}, \mathbf{r}^{\prime}} t_{\mathbf{r r}^{\prime}}^{\sigma} c_{\mathbf{r} \sigma}^{\dagger} c_{\mathbf{r}^{\prime} \sigma}
$$

$$
t_{\mathbf{r r}}^{\sigma}= \begin{cases}4 t+U(\mathbf{r}), & \mathbf{r}=\mathbf{r}^{\prime} \\ -t \pm i \sigma(\alpha / 2 a) u_{y}\left[\left(\mathbf{r}+\mathbf{r}^{\prime}\right) / 2\right], & \mathbf{r}=\mathbf{r}^{\prime} \pm a \mathbf{e}_{x} \\ -t \mp i \sigma(\alpha / 2 a) u_{x}\left[\left(\mathbf{r}+\mathbf{r}^{\prime}\right) / 2\right], & \mathbf{r}=\mathbf{r}^{\prime} \pm a \mathbf{e}_{y},\end{cases}
$$

where $c_{\mathbf{r} \sigma}$ is the annihilation operator of an electron at position $\mathbf{r}$ with spin $\sigma$, and $t=\hbar^{2} / 2 m a^{2}$ is the hopping energy. In what follows we regard each arm of the cross as the lead maintained at a different chemical potential $\mu_{\lambda}$ and the central square intersection of the cross as the sample region being in a nonequilibrium steady-state. 
The retarded (advanced) Green's function for the sample region is expressed as

$$
G_{\sigma}^{R(A)}=\left(E-H_{\sigma}-\sum_{\lambda} \Sigma_{\lambda \sigma}^{R(A)}\right)^{-1},
$$

with $\Sigma_{\lambda \sigma}^{R(A)}$ being the retarded (advanced) self-energy due to lead $\lambda$. The self-energy is generally represented in the form $\Sigma=V G V^{\dagger}$, where $G$ is the surface Green's function of the isolated semi-infinite lead, and $V$ is the hopping matrix that connects two neighboring transverse slices. The surface Green's function is obtained by numerically solving the quadratic matrix equation $G=G_{0}+G_{0} V G V^{\dagger} G$ with the Green's function of the single slice $G_{0} \cdot{ }^{32-34}$ In the nonequilibrium Green's function formalism, ${ }^{20,21}$ the density matrix for the sample region is expressed with the spectral function $A_{\lambda \sigma}(\varepsilon)=G_{\sigma}^{R}(\varepsilon) \Gamma_{\lambda \sigma}(\varepsilon) G_{\sigma}^{A}(\varepsilon)$ as

$$
n_{\mathrm{rr}^{\prime}}^{\sigma}=\left\langle c_{\mathbf{r}^{\prime} \sigma}^{\dagger} c_{\mathbf{r} \sigma}\right\rangle=\frac{1}{2 \pi} \sum_{\lambda} \int_{-\infty}^{\infty} d \varepsilon f\left(\varepsilon-\mu_{\lambda}\right)\left[A_{\lambda \sigma}(\varepsilon)\right]_{\mathbf{r r}^{\prime}}
$$

where $\Gamma_{\lambda \sigma}=i\left(\sum_{\lambda \sigma}^{R}-\sum_{\lambda \sigma}^{A}\right)$, and $f\left(\varepsilon-\mu_{\lambda}\right)$ is the Fermi-Dirac distribution function in lead $\lambda$ through which the chemical potential $\mu_{\lambda}$ relates to the density matrix. ${ }^{4,35-39}$ For a small variation of chemical potential $\delta \mu_{\lambda}$, the deviation of $n_{\mathbf{r r}^{\prime}}^{\sigma}$ from its equilibrium value at zero temperature is simply given by $\delta n_{\mathbf{r r}^{\prime}}^{\sigma}=(1 / 2 \pi) \Sigma_{\lambda} \delta \mu_{\lambda}\left[A_{\lambda \sigma}(E)\right]_{\mathbf{r r}}$, where $E$ is the equilibrium Fermi energy. The density matrix is directly used to evaluate the nonequilibrium distributions of spin density as well as spin current density whose operators are written in the bilinear form. ${ }^{4,35,37}$ In our lattice model they are explicitly represented as

$$
\begin{gathered}
\rho_{s}(\mathbf{r})=\frac{\hbar}{2 a^{2}} \sum_{\sigma} \sigma n_{\mathbf{r r}}^{\sigma}, \\
\mathbf{J}_{s}(\mathbf{r})=\frac{t}{2 a} \sum_{\sigma} \sum_{ \pm} \sigma\left( \pm \operatorname{Im} n_{\mathbf{r} \pm \mathbf{a}, \mathbf{r}}^{\sigma} \mathbf{e}_{x} \pm \operatorname{Im} n_{\mathbf{r} \pm \mathbf{b}, \mathbf{r}}^{\sigma} \mathbf{e}_{y}\right) \\
+\frac{\alpha}{4 a^{2}} \sum_{\sigma} \sum_{ \pm}\left\{u_{y}(\mathbf{r} \pm \mathbf{a} / 2) \operatorname{Re} n_{\mathbf{r} \pm \mathbf{a}, \mathbf{r}}^{\sigma} \mathbf{e}_{x}\right. \\
\left.-u_{x}(\mathbf{r} \pm \mathbf{b} / 2) \operatorname{Re} n_{\mathbf{r} \pm \mathbf{b}, \mathbf{r}}^{\sigma} \mathbf{e}_{y}\right\},
\end{gathered}
$$

with $\mathbf{a}=a \mathbf{e}_{x}$ and $\mathbf{b}=a \mathbf{e}_{y}$. These expressions also describe $\delta \rho_{s}(\mathbf{r})$ and $\delta \mathbf{J}_{s}(\mathbf{r})$ by simply replacing $n_{\mathrm{rr}^{\prime}}^{\sigma}$ with $\delta n_{\mathbf{r r}^{\prime}}^{\sigma}$. The local continuity of spin current $\nabla \cdot \mathbf{J}_{s}(\mathbf{r})=0$ in the steady-state is ensured at the operator level by the Heisenberg equation for spin density. The spin-resolved current flowing through the lead into the sample region is described by the linearized Landauer-Büttiker formula

$$
I_{\lambda \sigma}=\frac{e^{2}}{h} \sum_{\lambda^{\prime}} T_{\lambda \lambda^{\prime}}^{\sigma}\left(V_{\lambda}-V_{\lambda^{\prime}}\right),
$$

where $T_{\lambda \lambda^{\prime}}^{\sigma}=\operatorname{Tr}\left(\Gamma_{\lambda \sigma^{\prime}} A_{\lambda^{\prime} \sigma}\right)$ is the transmission coefficient from lead $\lambda^{\prime}$ to lead $\lambda$, and $V_{\lambda}$ is the voltage of lead $\lambda .{ }^{20}$ The charge current and the spin current are expressed as $I_{\lambda}^{c}$ $=\Sigma_{\sigma} I_{\lambda \sigma}$ and $I_{\lambda}^{S}=(\hbar / 2 e) \Sigma_{\sigma} \sigma I_{\lambda \sigma}$, respectively. It is shown from
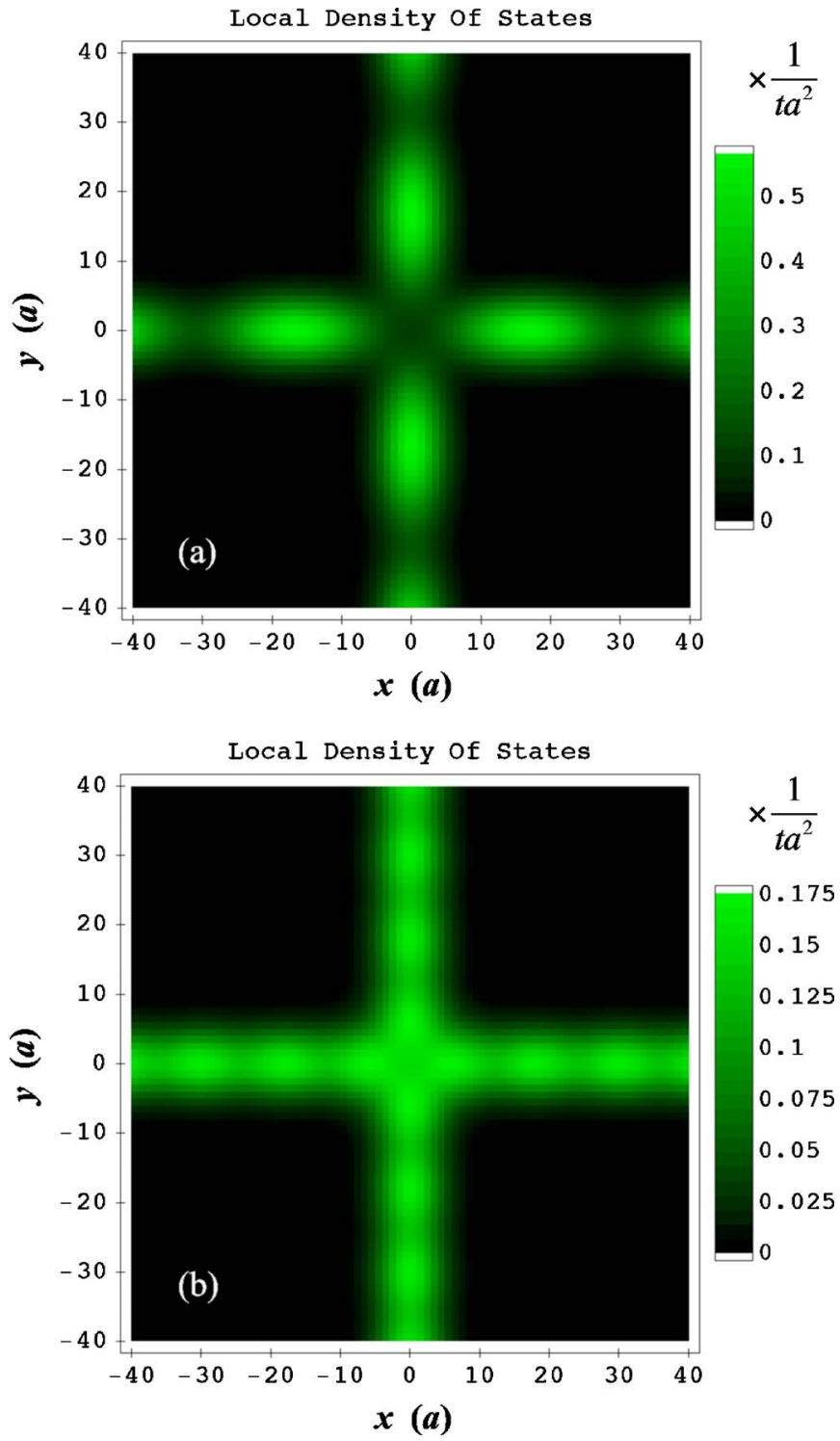

FIG. 2. (Color online) Local density-of-states $N(\mathbf{r}, E)$ calculated for $W=80 a$ at the Fermi energies $E=0.619 \hbar \omega$ (a) and $E=1.18 \hbar \omega$ (b), which correspond to the first peak and valley of spin Hall conductance $G_{\mathrm{SH}}$, respectively.

time-reversal and geometrical symmetries of the present system that $I_{2}^{c}=I_{4}^{c}=0$ and $I_{1}^{s}=I_{3}^{s}=0$ when $V_{2}=V_{4}=\left(V_{1}+V_{3}\right) / 2$. The longitudinal charge conductance is then defined as $G_{C}$ $=I_{1}^{c} /\left(V_{1}-V_{3}\right)$ for a pure charge current $I_{1}^{c}=-I_{3}^{c}$, and simultaneously the spin Hall conductance as $G_{\mathrm{SH}}=I_{4}^{s} /\left(V_{1}-V_{3}\right)$ for a pure spin current $I_{4}^{S}=-I_{2}^{S}$.

In our calculations, the energy $\hbar \omega$ is taken as the energy unit. The hopping energy is normally set at $t=10 \hbar \omega$, which corresponds to the length scale of the confinement $l \cong 5 a$, permitting our tight-binding model to reasonably simulate a continuum system. This has been ensured from the calculations with varying the ratio $l / a$. Figure 1 shows the longitudinal charge conductance $G_{C}$ and the spin Hall conductance $G_{\mathrm{SH}}$ calculated as a function of the Fermi energy $E$ ranging from 0 to $10 \hbar \omega$ for the normalized SO coupling strength $\alpha / l^{2}=0.1$ and a system size $W=60 a$. The charge conductance 

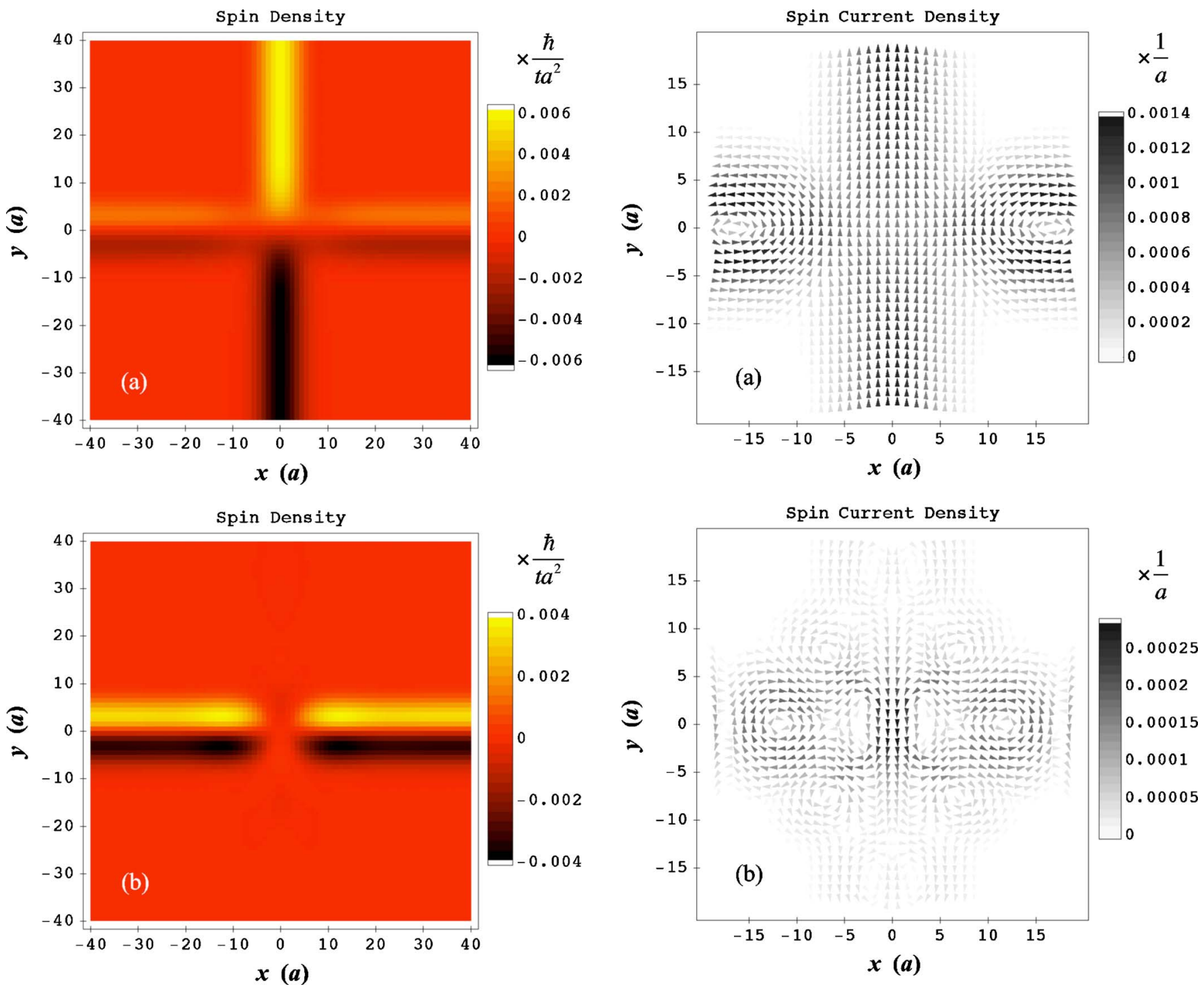

FIG. 3. (Color online) Normalized spin density $\delta \rho_{s}(\mathbf{r}) / \delta \mu$ for $W=80 a$. In the calculation, the deviations of chemical potentials were assumed to be $\delta \mu / 2=\delta \mu_{3}=-\delta \mu_{1}>0$ and $\delta \mu_{2}=\delta \mu_{4}=0$ for four leads. The Fermi energies are $E=0.619 \hbar \omega$ (a) and $E=1.18 \hbar \omega(\mathrm{b})$.

$G_{\mathrm{C}}$ is not fully quantized but exhibits a series of steps that are characteristic of ballistic 1D quantum channels. The onset of $G_{\mathrm{C}}$ steps is very close to the bottom of the 1D subband $(n+1 / 2) \hbar \omega$ for a single $\mathrm{QW}$, particularly in the low energy region. A sharp dip just below the onset accompanies the $G_{\mathrm{C}}$ steps. The spin conductance $G_{\mathrm{SH}}$ oscillates with the Fermi energy. The $G_{\mathrm{SH}}$ peak appears above the onset of $G_{\mathrm{C}}$ steps while the valley below the $G_{\mathrm{C}}$ dips. For $V_{1}>V_{3}$, electrons travel from the right lead to the left lead and then the SO coupling force pushes a spin- $\uparrow$ electron upward and a spin- $\downarrow$ electron downward. The positive sign of $G_{\mathrm{SH}}$ agrees with this interpretation. No significant system-size dependencies of $G_{\mathrm{C}}$ and $G_{\mathrm{SH}}$ are expected as long as the Fermi energy is sufficiently low that the relevant current-carrying states are hardly affected by the surrounding hard wall boundaries. The result presented in Fig. 1, for which the value of the confin-

FIG. 4. Normalized spin current density $\delta \mathbf{J}_{s}(\mathbf{r}) / \delta \mu$ for $W=40 a$. The set of chemical potentials is the same as that in Fig. 3. The Fermi energies are $E=0.619 \hbar \omega$ (a) and $E=1.18 \hbar \omega(\mathrm{b})$.

ing potential $U$ exceeds $20 \hbar \omega$ at the borders, is essentially the same as those calculated for larger systems. The SO coupling strength is thought to be the most fundamental parameter for the spin Hall effect. We have also investigated the variation of $G_{\mathrm{SH}}$ with the SO coupling strength in the range $0<\alpha / l^{2}<1$. The results of the calculations show that $G_{\mathrm{SH}}$ increases linearly with $\alpha / l^{2}$ up to $\alpha / l^{2} \cong 0.2$ and then tends to saturate.

The quenching of longitudinal charge current through the QW junction is due to the presence of a virtual or resonant state that is localized near the center of the junction and extends its wave function towards all four arms of the cross. ${ }^{31,40,41}$ The resonant states bring about an enhanced scattering of electrons at the junction and, hence, generate the dips in $G_{C}$. Such a resonant electron scattering may largely diminish the SO coupling force as well as the spin Hall current. Figure 2 compares the spatial profiles of local density-of-states $N(\mathbf{r}, E)=-\left(1 / \pi a^{2}\right) \operatorname{Im} \Sigma_{\sigma}\left[G_{\sigma}^{R}(E)\right]_{\mathbf{r r}} \quad$ calculated for $W=80 a$ at the first peak $(E=0.619 \hbar \omega)$ and valley 
$(E=1.18 \hbar \omega)$ of $G_{\mathrm{SH}}$. The formation of a resonant state is inferred from four bumps gathering near the center observed for the valley, which are distinct from a hollow observed for the peak. In the calculation, we have observed that these four bumps are merged into a single dominant peak at the subsequent $G_{C}$ dip. (This dip lies at $E=1.49 \hbar \omega$ but is too small to be seen in Fig. 1.) It is therefore plausible that the junction resonance causes the disappearance of spin Hall current around the specific Fermi energies.

The nonequilibrium distributions of spin density $\delta \rho_{s}$ and the spin current density $\delta \mathbf{J}_{s}$ are displayed in Figs. 3 and 4, respectively. In the calculation, the deviations of chemical potentials are chosen as $\delta \mu_{3}=-\delta \mu_{1}>0$ and $\delta \mu_{2}=\delta \mu_{4}=0$. The transverse spin separation driven by the longitudinal charge current is clearly visible in the figure. It is notable that the spin accumulation occurs uniformly, reflecting spin conservation during transport. For the $G_{\mathrm{SH}}$ peak, the spin population extending toward the top lead with a positive sign and the bottom lead with a negative sign is produced by the spin Hall current flowing upward. The spin current circulating near contacts with the right and the left leads occurs to satisfy the condition $I_{1}^{s}=I_{3}^{s}=0$. On the other hand, for the $G_{\mathrm{SH}}$ valley, the spin polarization disappears locally around the center of the sample and the uniform vertical flow of spin current is strongly suppressed. They are ascribed to the junction resonance discussed above.
Finally, we consider the magnitude of spin Hall conductance in realistic systems. The SO coupling strengths have been theoretically evaluated for some III-V semiconductor compounds. ${ }^{42}$ For example, $\alpha=4.4 \AA^{2}$ for GaAs, $110 \AA^{2}$ for InAs, and $500 \AA^{2}$ for InSb. Assuming a QW formed with $\hbar \omega$ of a few $\mathrm{meV},{ }^{27-29}$ the present calculation gives the estimates for $G_{\mathrm{SH}} \cong 10^{-4}-10^{-2}$ in units of $|e| / 4 \pi$ for these materials, which is rather small as compared with $G_{\mathrm{SH}} \cong 10^{-1}$ expected for the Rashba spin Hall effect in mesoscopic finite-size systems. ${ }^{8,34}$ Nevertheless, a unique property should be emphasized, namely that spin conservation is preserved in a QW system where the spin Hall effect exists, exemplifying the possibility of spin manipulation without spin-mixing processes in conventional semiconductors.

In summary, we studied the SO coupling in 2D electron systems with an in-plane potential gradient and its application to spin Hall effect. In such systems, electron spin is conserved whereas a SO coupling force exists that tends to separate electrons with different spins when they are moving. The existence of transverse spin separation and the spin Hall current in response to the longitudinal charge current are confirmed by numerical calculation for a four-terminal junction of harmonic QWs in the clean limit. The calculation also reveals the oscillation of spin Hall current with Fermi energy, which is accounted for by the junction resonance.
${ }^{1}$ I. Zutic, J. Fabian, and S. D. Sarma, Rev. Mod. Phys. 76, 323 (2004).

${ }^{2}$ J. E. Hirsch, Phys. Rev. Lett. 83, 1834 (1999).

${ }^{3}$ S. Murakami, N. Nagaosa, and S.-C. Zhang, Phys. Rev. B 69, 235206 (2004).

${ }^{4}$ M. Onoda and N. Nagaosa, Phys. Rev. Lett. 95, 106601 (2005).

${ }^{5}$ X.-L. Qi, Y.-S. Wu, and S.-C. Zhang, cond-mat/0505308 (to be published).

${ }^{6}$ J. Sinova, D. Culcer, Q. Niu, N. A. Sinitsyn, T. Jungwirth, and A. H. MacDonald, Phys. Rev. Lett. 92, 126603 (2004).

${ }^{7}$ E. M. Hankiewicz, L. W. Molenkamp, T. Jungwirth, and J. Sinova, Phys. Rev. B 70, 241301 (2004).

${ }^{8}$ B. K. Nikolic, L. P. Zarbo, and S. Souma, Phys. Rev. B 72, 075361 (2005).

${ }^{9}$ L. Sheng, D. N. Sheng, and C. S. Ting, Phys. Rev. Lett. 94, 016602 (2005).

${ }^{10}$ L. Sheng and C. S. Ting, Physica E (Amsterdam) 33, 216 (2006).

${ }^{11}$ C. L. Kane and E. J. Mele, Phys. Rev. Lett. 95, 226801 (2005).

${ }^{12}$ L. Sheng, D. N. Sheng, C. S. Ting, and F. D. M. Haldane, Phys. Rev. Lett. 95, 136602 (2005).

${ }^{13}$ B. A. Bernevig and S.-C. Zhang, Phys. Rev. Lett. 96, 106802 (2006).

${ }^{14}$ Y. A. Bychkov and E. I. Rashba, J. Phys. C 17, 6039 (1984).

${ }^{15}$ S.-Q. Shen, Phys. Rev. Lett. 95, 187203 (2005).

${ }^{16}$ B. K. Nikolic, L. P. Zarbo, and S. Welack, Phys. Rev. B 72, 075335 (2005).

${ }^{17}$ B. Zhou, L. Ren, and S.-Q. Shen, Phys. Rev. B 73, 165303 (2006).

${ }^{18}$ K. Y. Bliokh, cond-mat/0511146 (to be published).
${ }^{19}$ A. Berard and H. Mohrbach, Phys. Lett. A 352, 190 (2006).

${ }^{20}$ S. Datta, Electronic Transport in Mesoscopic Systems (Cambridge University Press, Cambridge, 1995).

${ }^{21}$ G. D. Mahan, Many-Particle Physics (Kluwer Academic/Plenum, New York, 2000).

${ }^{22}$ S. Bellucci and P. Onorato, Phys. Rev. B 73, 045329 (2006).

${ }^{23}$ Q.-F. Sun and X. C. Xie, Phys. Rev. B 72, 245305 (2005).

${ }^{24}$ J. Shi, P. Zhang, D. Xiao, and Q. Niu, Phys. Rev. Lett. 96, 076604 (2006).

${ }^{25}$ N. Sugimoto, S. Onoda, S. Murakami, and N. Nagaosa, Phys. Rev. B 73, 113305 (2006).

${ }^{26}$ A. V. Moroz and C. H. W. Barnes, Phys. Rev. B 60, 14272 (1999)

${ }^{27}$ S. E. Laux, D. J. Frank, and F. Stern, Surf. Sci. 196, 101 (1988).

${ }^{28}$ H. Drexler, W. Hansen, S. Manus, J. P. Kotthaus, M. Holland, and S. P. Beaumont, Phys. Rev. B 49, 14074 (1994).

${ }^{29}$ B. Kardynal, C. H. W. Barnes, E. H. Linfield, D. A. Ritchie, J. T. Nicholls, K. M. Brown, G. A. C. Jones, and M. Pepper, Phys. Rev. B 55, R1966 (1997).

${ }^{30}$ Recently, it is suggested that the SO coupling induced by the lateral confining potential, particularly a parabolic one, causes the opposite spin accumulations on the transverse two edges of the finite-size $2 \mathrm{D}$ system under the longitudinal voltage bias as a possible mechanism to interpret spin Hall experiments. See Y. Xing, Q.-F. Sun, L. Tang, and J.-P. Hu, cond-mat/0604502 (to be published); Y. Jiang and L. Hu, Phys. Rev. B 74, 075302 (2006). The physics involved in this phenomenon can be easily understood in terms of the spin-dependent SO coupling force and the resulting eigenfunction displacement described in the present paper. 
${ }^{31}$ G. Kirczenow, Phys. Rev. Lett. 62, 2993 (1989).

${ }^{32}$ N. J. Higham and H.-M. Kim, IMA J. Numer. Anal. 20, 499 (2000).

${ }^{33}$ S. Rotter, B. Weingartner, N. Rohringer, and J. Burgdörfer, Phys. Rev. B 68, 165302 (2003).

${ }^{34}$ J. Wang and K. S. Chan, Phys. Rev. B 72, 045331 (2005).

${ }^{35}$ M. Onoda and N. Nagaosa, Phys. Rev. B 72, 081301 (2005).

${ }^{36}$ B. K. Nikolic, S. Souma, L. P. Zarbo, and J. Sinova, Phys. Rev. Lett. 95, 046601 (2005).

${ }^{37}$ B. K. Nikolic, L. P. Zarbo, and S. Souma, Phys. Rev. B 73, 075303 (2006).
${ }^{38}$ J. Wang, K. S. Chan, and D. Y. Xing, Phys. Rev. B 73, 033316 (2006).

${ }^{39}$ A. Reynoso, G. Usaj, and C. A. Balseiro, Phys. Rev. B 73, 115342 (2006).

${ }^{40}$ R. L. Schult, D. G. Ravenhall, and H. W. Wyld, Phys. Rev. B 39, 5476 (1989).

${ }^{41}$ D. G. Ravenhall, H. W. Wyld, and R. L. Schult, Phys. Rev. Lett. 62, 1780 (1989).

${ }^{42}$ E. A. de Andrada e Silva, G. C. La Rocca, and F. Bassani, Phys. Rev. B 55, 16293 (1997). 